\title{
On resolving the crisis in particle physics associated with the fall of supersymmetry
}

\author{
Volodymyr Krasnoholovets \\ Institute of Physics, National Academy of Sciences of Ukraine, \\ Prospect Nauky 46, UA-03028 Kyiv, Ukraine \\ e-mail: krasnoh@iop.kiev.ua
}

\begin{abstract}
Classical mechanics of a particle was gradually complicated and expanded by adding new and new attributes, which finally brought to the situation that in principle cannot be verified experimentally. Here, a possible scenario for the further development of particle physics is suggested, which is based on a deterministic submicroscopic concept that has been developing by the author.
\end{abstract}

\section{Provisions of particle physics related to supersymmetry}

In classical mechanics the state of a particle is determined exactly and all observables take exact values. In the gravitation physics an important role plays spacetime, which in the formalism of general relativity became a smooth manifold with a pseudo-Riemannian metric

$d s^{2}=\sum_{\mu \nu} g_{\mu v} d x^{\mu} d x^{V}$ of signature (+, -, -, -). Such an approach made it possible to talk about the gravitation as a purely geometric phenomenon, a manifestation of the curvature of spacetime. Einstein interpreted the $g_{\mu v}$ as the gravitational potentials and showed that in matter-free regions of spacetime they satisfy $R_{i j}=0$ where $\mathrm{R}$ are the components of the Ricci tensor. These are the Einstein equations. Unlike the Maxwell equations they are nonlinear in the $g_{\mu v}$

Contrary to the gravitation physics, quantum physics uses the mathematical model in which the states are believed causal and deterministic until measurements are not made. This is the Copenhagen interpretation. One of the triumphs of quantum theory and the Copenhagen interpretation was an introduction of the wave-particle duality of light; in particular, such interpretation was used to explain the phenomenon of the diffraction of light [1,2].

The development of mathematics associated with quantum phenomena and the introduction of metric of spacetime was consistently described by Varadarajan [3]. A quantum system is described mathematically by using a complex separable Hilbert space $H$. The states of the system are the points of the projective space $P(H)$ of $H$. In a one-dimensional subspace of $H$ there is a basis vector $\psi$ of norm 1 . If the observable (operator) $A$ has a discrete spectrum with eigenvalues $\mathrm{A} 1, \mathrm{~A} 2, \ldots$ and corresponding (unit) eigenvectors $\psi_{1}, \psi_{2}, \ldots$ Then a measurement of $A$ in the state $\psi$ will give the value $A i$ with probability $\left|\psi, \psi_{i}\right|^{2}$ where $\mathrm{i}=1,2, \ldots$ The complex number $\left(\psi, \psi_{i}\right)$ is called the probability amplitude, so that quantum probabilities are computed as squares of absolute values of complex probability amplitudes. Since basis vectors $\psi_{i}$ are orthonormal basis of $H$, the following normalization relationship is hold: $\sum_{i}\left|\left(\psi, \psi_{i}\right)\right|^{2}=1$

A special property revealed in particles was named „spin ${ }^{e e}$. From the mathematical point of view, spin systems of $\mathrm{N}$ particles are systems in which all observables have exactly $\mathrm{N}$ values. The Hilbert space can then be taken to be $\mathrm{C}^{N}$ with the standard scalar product. The observables are then $\mathrm{N}$ x $\mathrm{N}$ Hermitian matrices whose spectra are the sets of values of these observables. The determination of states is made by measurements of observables with exactly $\mathrm{N}$ distinct values.

Although the quantum observables do not form an algebra, they are the real elements of a complex algebra. Thus one can say that the transition from classical to quantum theory is achieved by replacing the commutative algebra of classical observables by a complex algebra with involution whose real elements form the space of observables of the quantum system. In quantum theories the basic role belongs to the commutation rule between position and momentum of a particle: 


$$
[p, q]=-i \hbar
$$

If the quantum of action, Planckees constant ${ }^{\text {ee }}$, puts equal to zero, the commutation goes over to zero, $[\mathrm{p}, \mathrm{q}]=0$, which corresponds to the classical case.

Therefore a really deeper study of quantum foundations based on relationship (1) started by using the noncommutative quantum observable algebra. Quantization, by which the transition from a classically described system to a ,corresponding quantum system," is a deformation of the classical commutative algebra into a noncommutative quantum algebra. The notion of „quantization is specified into a defined mathematical operation.

The properties of elementary particles and the structure of Lie groups and Lie algebras allowed physicists to give rise to an irreducible representation of the Poincaré group of different quantum states of an elementary particle. Space translations, time translations, rotations, and boosts, all taken together, constitute the Poincare group. The group elements are the three rotation matrices and three boost matrices (as in the Lorentz group), and one for time translations and three for space translations in spacetime. The properties of the various particles, in particular their spectra, are related to representations of Lie algebras, corresponding to "approximate symmetries" of the universe.

The next important property in the mathematical description of quantum systems is the notion of their symmetry. Symmetries in quantum mechanics describe features of spacetime and particles, which are unchanged under some quantum mechanical transformation. In general, symmetry in physics, invariance, and conservation laws, are fundamentally important constraints for formulating physical theories and models. Symmetries are bijections of the space of states preserving measurement probabilities [3]; by the fundamental theorem of projective geometry these are therefore given by $\phi \mapsto U \phi$ where $U$ is unitary or antiunitary: $(U \phi, U \psi)=(\phi, \psi)$ or $(U \phi, U \psi)=(\psi, \phi)$. There are internal symmetries, spacetime symmetries and gauge symmetries.

For example, the element of SU(2) that describes the internal symmetry is allowed to depend on the spacetime point where the particle is located; these local symmetries are then described by functions on spacetime with values in SU(2) [3]. They are called gauge symmetries, and the group of all such (smooth) functions is called the gauge group.

The so-called isospin symmetry is also characterised by $\mathrm{SU}(2)$ group corresponding to the similarity between up quarks and down quarks. Similarly, a proton can be transformed into a neutron, or into a superposition of a proton and neutron, but not into any other particles. Therefore, the transformations move the proton around a two-dimensional space of quantum states. The proton and neutron are called an "isospin doublet", mathematically analogous to how a spin-1/2 particle behaves under ordinary rotation.

When these unitary transformations are applied to any of the three pions $\left(\pi^{0}, \pi^{+}\right.$, and $\left.\pi^{-}\right)$, it can change any of the pions into any other, but not into any non-pion particle. Therefore, the transformations move the pions around a three-dimensional space of quantum states. The pions are called an "isospin triplet", mathematically analogous to how a spin-1 particle behaves under ordinary rotation.

Researchers also consider the so-called symmetry breaking that is describing a phenomenon where small fluctuations, which act on a system at a critical point, determine the system 's fate, i.e. which branch of a bifurcation is taken. In a physical system at spontaneous symmetry breaking the underlying laws remain invariant under a symmetry transformation, though the system changes under the transformation, in contrast to explicit symmetry breaking. As the result, a system is transformed from a symmetrical state to an asymmetrical one.

Bosons with integral spin and fermions with half-integral spin obey different statistics - BoseEinstein (the trivial representation) and Fermi-Dirac (the signature representation), respectively.

The spin states of a particle form an irreducible module for $\mathrm{SU}(2)$ of dimension $2{ }_{j}+1$ where 
$j \in \frac{1}{2} \mathbf{Z}$ is the spin. Particles with $\mathbf{j}$ integral (integral spin) are bosons and with half-integral are fermions (half-integral spin). In a quantum field theory that contains interacting particles of both spin parities, the Hilbert space $H$ of 1-particle states has a decomposition

$$
H=H_{0} \otimes H_{1}
$$

Where $H_{0}$ is the space of states where there is one boson and $H_{1}$ is the space of states where there is one fermion. For the N-particle space the Hilbert space becomes

$$
H_{N}=\bigotimes_{1 \leq d \leq N} S^{d}\left(H_{0}\right) \otimes \Lambda^{N-d}\left(H_{1}\right)
$$

Where $S^{d}\left(H_{0}\right)$ is the space of symmetric tensors (corresponds to the Bose-Einstein statistics) and $\Lambda^{N-d}\left(H_{1}\right)$ is the space of antisymmetric tensors (corresponds to the Fermi-Dirac statistics). Thus the physicists suggested do not treat separately the bosonic and fermionic cases and the unified consideration would result in increased clarity and understanding. Such a unified theory is a linear theory where all (linear) objects are systematically graded by Z2, just as the Hilbert space of 1- particles above was graded into bosonic and fermionic parts. Infinitesimal symmetry of such graded spaces is viewed as a type of symmetry that sends bosonic states into fermionic states and vice versa. The super commutator is defined by

$$
[a, b]= \begin{cases}a b-b a & \text { if at least one of } a, b \text { is even } \\ a b+b a & \text { if both } a, b \text { are odd }\end{cases}
$$

These supersymmetries obey a Grassmann, or exterior algebra in which the Fermi fields comply not commutation rules but anticommutation rules. There appeared supersymmetric quantum field theories, gauge theories and a supersymmetric extension of Einstein ${ }^{\text {ee }}$ s theory of gravitation to supergravity, which motivated researchers for seeking a unified field theory.

Each super particle, when viewed as a unitary representation of the underlying Poincare group, is the direct sum of a collection of ordinary particles, called a multiplet. The members of a multiplet are called partners of each other. For a boson there is a superpartner fermion called a bosino and for each fermion, there is a superpartner boson called a sfermion: electron - selectron, neutrino sneutrino, quark - squark, photon - sphoton, graviton - gravitino, $W^{ \pm}-$Wino $^{ \pm}$, etc. In such an approach the geometry of spacetime is described locally by a set of coordinates consisting of the usual ones, which in addition are supplemented by a set of anticommuting (Grassmann) coordinates. String theory and membranes consider objects as not point-like but extended. Thus spacetime geometry at the Planck scale becomes almost surely non-commutative (as there are no points). At the same time, the supersymmetric approach did not allow one to construct a convincing noncommutative geometrical theory that has the Riemann-Einstein geometry as a limit.

However, notwithstanding the so remarkable theories developed for about 50 years, particle experiments still do not present any proof of the existence of superpartners for known particles. That is why particle physicists state about a crisis in physics. Shifman [4] called on his colleagues to stop to modify supersymmetry, as the theory has failed experimental tests; he points out it is time to 
start thinking and developing new ideas. Lykken and Spiropulu pronounce: "Yet if superpartners are not found, we face a paradigm rupture in our basic grasp of quantum physics" [5]. Thus, if supersymmetry does not pan out, scientists need a new way to explain the universe.

\section{What particle physicists do not take into account?}

Let us consider major statements accepted by quantum physics at face value without a thorough check of their conception and correctness.

First of all the commutation rule (1) is transferred to the geometry of space as the most fundamental property of the microcosm. This relationship uses as basic at the construction of noncommutative geometries. But what is hidden behind such a strange property of the microcosm? This question is out of interest of particle physicists.

Second, the wave $\psi$-function is extremely important in quantum mechanics and quantum field theories, however, what properties of a particle does it represent exactly? Max Born ascribed it statistical properties - the module $|\psi(\vec{r})|$ describes the probability of a particle being at the point $\vec{r}$. .However, Louis de Broglie insisted that the $\psi(\vec{r})$ should be associated with real physical characteristics of the appropriate particle.

Third, the notion of a wave-particle is still mystical. De Broglie did not support the unification of a moving particle with a wave; he was looking for the double solution theory in which the particle and its wave could be treated separately.

Forth, the notion of the Compton wavelength, which ascribed a radius of hardness of the particle in question, is complete ignored: particles are considered as point-like or as a one-dimensional abstract string with about 10 dimensions.

Fifth, the notion of spin: particle physicists are not interested in the investigation of this phenomenon in detail; they ignore studies of other researchers who try to understand the phenomenon. Nevertheless, the presence of spin in particles is considered as the most essential characteristic of particles in the formation of the theory of supersymmetry.

Sixth, gravity is still described in the framework of a phenomenological approach and even quantum gravity starts from ideas of phenomenological formalism of general relativity. Quantum properties of the microcosm are rather ignored.

Seventh, dark matter and dark energy are out of the standard model, which directly points out to serious incompleteness of the model.

Eight, particle physics has never been interested in searching for the answers to such challenges as: what is mass, what is electric charge, what is photon, etc.?

Ninth, what is the principal difference between leptons and quarks, which interact very unlikly?

Tenth, space - what is it? What is the constitution of real physical space?

\section{A submicroscopic concept}

The standard model of particle physics combines all fundamental interactions in a unified theory the theory of everything. However, it seems more reasonable to start from the theorem of something, which is able to clarify the basic notions of quantum physics listed in Section 2. At the study of elementary particles researchers use Lie algebra, group theory, topology, symmetry and other mathematical disciplines. Thus mathematics provides service to the studies in particle physics. On the other hand, mathematics itself is able to generate fundamental physics and we will demonstrate this below.

The appearance of physics can be done through a pure mathematical analysis of the notion of space. Any property of any given object should be consistent with the characteristics of the corresponding embedding space. An unknown space can be explored through scanning operators and examined with respect to such fundamental notions as measure, distance, dimensionality, fractality and topology. These notions were revised a broad sense of totally topologically ordered space by Michel Bounias and the author [6-9] with the purpose to analyse the constitution of ordinary physical space. 
One may start from nothing - a flat space that does not manifest itself. Since the original space is flat, it does not have Poisson brackets and any other forms of non-commutative features. The Jordan and Lebesgue measures involve respective mappings (I) and (M) on spaces which must be provided with operations $\cap, \cup$ and C. In spaces of the $\mathrm{R}^{\mathrm{n}}$ type, tessellation by balls is involved, which requires a distance for the measure of diameters of intervals. These intervals can be replaced by topological balls and the evaluation of their diameter needs an appropriate general definition of a distance. A space $\mathrm{E}$ is ordered if any segment owns an infinum and a supremum. That is why a distance $\mathrm{d}$ between $\mathrm{A}$ and $\mathrm{B}$ is represented by the relation

$$
d(\mathrm{~A}, \mathrm{~B}) \subseteq \operatorname{dist}(\inf \mathrm{A}, \inf \mathrm{B}) \cap \operatorname{dist}(\sup \mathrm{A}, \operatorname{supB})
$$

with the distance evaluated through either classical forms or even the set-distance $\Delta(\mathrm{A}, \mathrm{B})$. Any topological space is metrizable as provided with the set-distance $\Delta$ as a natural metrics. All topological spaces are kinds of metric spaces called "delta-metric spaces". Distance $\Delta(\mathrm{A}, \mathrm{B})$ is a kind of an intrinsic case $\left[\Lambda_{(A, B)}(A, B)\right]$ of $\Lambda_{E}(A, B)$ while the latter is called a "separating distance". The separating distance also stands for a topological metrics. Hence, if a physical space is a topological space, it will always be measurable.

A fundamental segment $(A, B)$ and intervals $L_{i}=\left[A_{i}, A_{(i+1)}\right]$ allow one to determine similarity coefficients for each interval by $\rho_{i}=\operatorname{dist}\left(\mathrm{A}_{i} ; \mathrm{A}_{(i+1)}\right)=\operatorname{dist}(\mathrm{A}, \mathrm{B})$.

The similarity exponent of Bouligand (e) is such that for a generator with $n$ parts:

$$
\sum_{i \in[1, n]}\left(\rho_{i}\right)^{\mathrm{e}}=1
$$

Then, when e is an integer, it reflects a topological dimension

$$
\mathrm{e} \approx \log n / \log \rho
$$

which means that a fundamental space $\mathrm{E}$ can be tessellated with an entire number of identical ballsB exhibiting a similarity with $E$, upon coefficient $r$

The measure of the size of tessellating balls as well as that of tessellated space, with reference to the calculation of their dimension is determined through relations (5), (6). A space may be composed of members, such that not all tessellating balls have identical diameter. Also a ball with two members would have a more complicated diameter. Hence a measure should be used as a probe for the evaluation of the coefficient of size ratio needed for the calculation of a dimension.

It is generally assumed that some set does exist. This strong postulate was reduced to the axiom of the existence of the empty set. It was shown that providing the empty set $(\varnothing)$ with $(\in \subset \subset)$ as the combination rules with the property of complementarity $(C)$ results in the definition of a magma without violating the axiom of foundation if the empty set is seen as a hyperset that is a nonwellfounded set.

It was proved that the magma $\varnothing^{\varnothing}=\{\varnothing, \mathbf{C}\}$ constructed with the empty hyperset and the axiom of availability is a fractal lattice. Writing $\varnothing^{\varnothing}$ denotes that the magma reflects the set of all selfmappings of $(\varnothing)$, which emphasizes the forthcoming results. The space constructed with the empty set cells is a Boolean lattice $S(\varnothing)$ and this lattice is provided with a topology of discrete space. The magma of empty hyperset is endowed with self-similar ratios.

Such a lattice of tessellation balls was named a tessel-lattice [6]. The magma of empty hyperset is a fractal tessel-lattice [7]. 
A tessel-lattice of empty set cells represents a primary substrate, which is a physical space. Spacetime is submitted by ordered sequences of topologically closed Poincaré sections of this primary space. These mappings are constrained to provide homeomorphic structures serving as frames of reference in order to account for the successive positions of any objects present in the system. Mappings from one to the next section involve morphisms of the general structures, standing for a continuous reference frame, and morphisms of objects present in the various parts of this structure. The combination of these morphisms provides space-time with the features of a nonlinear generalized convolution. Discrete properties of the lattice allow the prediction of scales at which microscopic to cosmic structures should occur.

The fundamental metric of space-time is represented as the extension of 3D coordinates up to the 4th dimension timeless space through convolution with the volume of space. Fractality of space manifests itself through changes in the dimension of geometrical structures: the dimension of a curve exceeds 1D and falls in the interval between 1D and 2D; for a volumetric object the dimension may lie between 3D and 4D.

In a degenerate state the size of a ball, which plays the role of a lattice ee cell, is associated with the Planck length $\ell_{\mathrm{P}}=\sqrt{\hbar G / c^{3}} \cong 1.616 \times 10^{-35} \mathrm{~m}$. Deformations of primary cells by exchange of empty set cells allow a cell to be mapped into an image cell in the next section as far as mapped cells remain homeomorphic.

The tessel-lattice is specified with quanta of distances and quanta of fractality. The sequence of mappings of one into another structure of reference (e.g. elementary cells) represents an oscillation of any cell volume along the arrow of physical time.

Thus the tessel-lattice represents both relativistic space and quantic void: the concept of distance and the concept of time have been defined on it and such space holds for a quantum void (a vacuum) since it provides a discrete topology with quantum scales and on the other hand it contains no "solid" object that would stand for a given provision of physical matter.

When a fractal transformation is involved in the exchange of deformations between cells, a change in the dimension of the cell occurs and the homeomorphism is not conserved. The fractal kernel (a local deformation of the tessel-lattice) stands for a "particle" and the reduction of its volume is compensated by morphic changes of a finite number of surrounding cells.

A particle must be provided with physical properties. First of all this is mass: The mass $m_{A}$ of a particulate ball $\mathrm{A}$ is a function of the fractal-related decrease of the volume of the ball:

$$
m_{A} \propto\left(V^{\text {deg.cell }} / V^{\text {part }}\right) \cdot\left(\mathrm{e}_{\text {fract }}-1\right)_{\mathrm{e}_{\text {fract }}>1}
$$

Where $V^{\text {deg.cell }}$ is the typical average volume of a cell in the tessel-lattice in the degenerate state; $\mathrm{V}^{\text {part }}$ is the volume of the kernel cell of the particle; (e) is the Bouligand exponent, and (e fract -1$)$ the gain in dimensionality given by the fractal iteration. Just a volume decrease is not sufficient for providing a ball with mass, since a dimensional increase is a necessary condition (there should be a change in volumetric fractality of the ball).

Therefore, in the tessel-lattice a particle is a contracted kernel-cell. Surrounding cells compensate this local deformation by morphic changes, a typical tension of the surrounding tessel-lattice, whichforms a peculiar deformation coat with a radius identified with the particle ${ }^{e e}$ Compton wavelength $\lambda_{\mathrm{Com}}=h /(\mathrm{mc})$. This radius/diameter of hardness manifests itself in experiments on light scattering by particles, as was demonstrated by Compton in 1923 [10]. Note that the existence of a deformation coat is a typical situation for a crystal lattice in a solid when a foreign particle or isotope defect enters the solid.

When a massive particle moves, it pulls its deformation coat with it, i.e. the state of the coat migrates from cell to cell by a fractal relay mechanism. The interaction of a moving particle-like deformation with the surrounding lattice involves a fractal decomposition process. 
The remark of Poincaré [11] has so far still been overlooked by the majority of researchers: a particle, which moves in the ether, is surrounded with the ether excitations. In the tessel-lattice a particle interacts with the surrounding cells and generates some excitations, which is exactly in line with Poincaréee s idea. An appropriate Lagrangian that describes such motion in a simplified form looks as follows [12]

$$
L=\frac{1}{2} m \dot{x}^{2}+\frac{1}{2} \mu \dot{\chi}^{2}-\frac{1}{T} \sqrt{m \mu} \dot{x} \chi
$$

where $m, x$ are the mass and position of the particle and $\mu, \chi$ are the mass and position of the particlee s cloud of excitations, respectively; $1 / \mathrm{T}$ is the frequency of collisions between the particle and the cloud. These excitations were named inertons, because they represent inert properties of the particle (the particle ${ }^{e e}$ resistance at the particle ${ }^{e c}$ motion through the space).

The Euler-Lagrange equations derived on the basis of the Lagrangian (8) lead to the oscillating solution for the particle velocity

$$
\dot{x}=v_{0} \cdot(1-|\sin (\pi t / T)|)
$$

between the initial value $v_{0}$ and zero along each section $\lambda$ of the particle path. This spatial amplitude is determined as follows: $\lambda=v_{0} T$. For the cloud of inertons: $\Lambda=c T$. These two amplitudes become connected by means of relationship

$$
\Lambda=\lambda c / v_{0}
$$

The further analysis allows us to derive the following two relationships

$$
E=h v, \quad \lambda=h / p,
$$

Where $h$ is the minimal action of the periodic motion of a particle when the particle is guided solely by the tessel-lattice and any influence on the side of external fields or direct contacts with other physical systems does not disturb the particle. Here, $v=1 /(2 T)$ is the frequency of the particle oscillations along its path, $p=m v_{0}$ is the momentum of the particle and $\mathrm{E}$ is the kinetic energy of the particle.

On the other hand, relationships (11) for a particle were suggested by de Broglie in 1924, which are basic for quantum mechanics. De Broglie suggested that a phase wave with the wavelength should be linked to the particle with the momentum $\mathrm{p}$ by means of relationships (11). In his opinion the wave should guide the particle. So, we can see that the de Broglie wave is generated by Poincarées ether excitations, i.e. inertons surrounding the particle.

Relationships (11) allow one to derive the Schrödinger equation

$$
-\frac{\hbar^{2}}{2 m} \nabla^{2} \psi(r, t)+V(r) \psi(r, t)=E \psi(r, t) .
$$

The submicroscopic concept operates with the particle and the particlees cloud of inertons. Conventional quantum mechanics, which was evolved in an abstract phase space on an atom scale, 
works with the wave $\psi$-function. These two approaches can be combined, as the inerton cloud of an entity, which is associated with the entity ${ }^{\text {ee }}$ s field of inertia, is mapped into an abstract phase space of conventional quantum mechanics in the form of a "mysterious" wave $\psi$-function.

So the amplitude of spatial oscillations of the particle $\lambda$ appears in quantum mechanics as the de Broglie wavelength. The amplitude of the particle ees cloud of inertons becomes implicitly apparent through the availability of the wave $\psi$-function. Therefore, the physical meaning of the wave $\psi$ function becomes completely clear: it describes the range of space around the particle perturbed by the particlee s inertons.

When the speed $v_{0}$ of a particle is close to the speed of light $c$, the classical Lagrangian

$$
L=-m_{0} c^{2} \sqrt{1-v_{0}^{2} / c^{2}}
$$

is transformed to the form that describes an inner process of emission and absorption of inertons by the particle:

$$
L=-m_{0} c^{2} \sqrt{1-\frac{1}{m_{0} c^{2}}\left\{m_{0} \dot{x}^{2}+\mu \dot{x}^{2}-\frac{2 \pi}{T} \sqrt{m_{0} \mu}\left(x \dot{\chi}+v_{0} \chi\right)\right\}}
$$

All this occurs in the section equal to the particle ${ }^{\text {ee }}$ de Broglie wavelength $\lambda$. The Euler-Lagrange equations lead to the same solutions, as is the case of the non-relativistic Lagrangian (8).

The Dirac equation can be derived from the Lagrangian (14) if one introduces "something" additional into it. This "something" is an intrinsic motion that can be associated with proper particle pulsations [13]. Then an appropriate Hamiltonian has a new term $c^{2} \vec{\pi}_{\uparrow(\downarrow)}^{2}$ which previously was not taken into account. This term describes proper pulsations of the particle between a bean-like and spherical shape in the section of $\lambda$ :

$$
H_{\uparrow(\downarrow)}^{\text {particle total }}=\sqrt{c^{2} \vec{p}^{2}+c^{2} \vec{\pi}_{\uparrow(\downarrow)}^{2}+m_{0}^{2} c^{4}}
$$

The Hamiltonian (15) shows two possible projections of intrinsic pulsations of the particle: ahead and behind. Decomposing the square root in expression (15) one gets a two matrix. This is the inner reason why the Dirac equation possesses matrix components associated with the particle spin $S_{\uparrow(\downarrow)}^{z}= \pm \hbar / 2$ which in the presence of a magnetic induction B renormalizes the eigenvalue $\mathrm{E}$ of the particle to the quantity $E+e B S_{\uparrow(\downarrow)}^{z} / m$.

A fractal volumetric deformation of a topological ball has been associated with the notion of mass. A fractal surface deformation of a topological ball can be associated with notion of elementary electric charge [14].

So a cell of space whose surface is covered of N needles, can be described by a field vector $\vec{\varepsilon}_{n}$ ( $n$ is the needle number), and can be associated with the scalar function $\Phi_{n}(h) \propto h$, i.e. $\vec{\varepsilon}_{n}=-\nabla_{h} \Phi_{n}(h)$; so $\vec{\varepsilon}_{n}$ is a co-vector. The spike of each $n$th needle is able to deviate from its equilibrium position, i.e., the bending of the needle from its axis must not be ruled out. The value of the displacement decreases from the top to the base of the needle, the latter being fixed. Therefore this kind of motion can be related to a vector field (only the motion of a point is described by a vector) and can be designated as $\vec{A}_{n}$.

In conventional symbols the Lagrangian density of the electromagnetic field, which interacts with a charge, takes the form 


$$
L_{\text {el-magn }}=\frac{\varepsilon_{0}}{2 c^{2}} \dot{\varphi}^{2}+\frac{\varepsilon_{0}}{2} \dot{\vec{A}}^{2}+\varepsilon_{0} \dot{\varphi} \nabla \vec{A}+\frac{\varepsilon_{0} c^{2}}{2}(\nabla \times \vec{A})^{2}-\rho \varphi+\rho \overrightarrow{v_{0}} \vec{A}
$$

The Lagrangian density (16) with the members $\dot{\varphi}$ (which is absent in the classical Lagrangian for the electric charge) allows one to derive the Euler-Lagrange equations that culminate in the Maxwell equations for the scalar $\varphi$ and vector $\vec{A}$ potentials in the so-called d'Alambert configuration

$$
\begin{aligned}
& \nabla^{2} \varphi-\frac{1}{c^{2}} \frac{\partial^{2} \varphi}{\partial t^{2}}=\frac{\rho}{\varepsilon_{0}}, \\
& \nabla^{2} \vec{A}-\frac{1}{c^{2}} \frac{\partial^{2} \vec{A}}{\partial t^{2}}=-\frac{\rho v_{0}}{\varepsilon_{0} c^{2}} .
\end{aligned}
$$

At the elementary level gravity occurs when inertons emitted by a moving particle return to it. Evidently the elastic tessel-lattice has to push these inertons back to the centre of emission. This idea allows a detailed mathematical study and, moreover, it can be extended to macroscopic objects. In a condensed matter, inerton clouds of vibrating entities overlap forming a total inerton cloud of all the entities, which spreads far beyond the object.

Now let us consider the phenomenon of gravity. Since inertons are carriers of fragments of the particle ${ }^{\text {ee }}$ mass, they carry inert and gravitational properties of the particle. Hence the particle moves while disintegrating its mass. Inertons irradiated by the particle come back to it and return fragments of its mass and velocity. Such motion can be described by a new kind of a Lagrangian:

$$
L=-m_{0} c^{2} \cdot\left\{\text { submicrosøic mechanics }+\frac{T^{2}}{2 m_{0}^{2}} \dot{m}^{2}+\frac{T^{2}}{2 \Lambda^{2}} \dot{\vec{\xi}}^{2}-\frac{T}{m_{0}} \dot{m} \nabla \vec{\xi}\right\}^{\frac{1}{2}},
$$

Here, $\mathrm{T}$ is the same time period of collisions of the particle and its inerton cloud as above; $m(\vec{r}, t)$ is the current mass of the system of "particle + inerton cloud"; $\vec{\xi}(\vec{r}, t)$ is the variable that describes a local distortion of the tessel-lattice, which can be called a tension. In other words, we introduce a new variable for the particle, which means that the mass is not an absolute value but variable. Namely, we assume that in the section of $\lambda$ the particle ${ }^{\text {ee }}$ characteristics oscillate between two different properties: the speed oscillates between $v_{0}$ and zero; the stable alteration of the kernel cell oscillates between the deformation $m$ and the tension $\xi$ which can be considered as oscillation of the mass between $m$ and zero and the oscillation of the tension between zero and $\xi$

The Euler-Lagrange equations for variables $m$ and $\vec{\xi}$ allow the solution in the form of standingspherical waves, which exhibit the dependence $1 / r$,

$$
\begin{aligned}
& m(r, t)=C_{1} \frac{m_{0}}{r} \cos \frac{\pi r}{2 \Lambda}\left|\cos \frac{\pi t}{2 T}\right| \\
& \xi(r, t)=C_{2} \frac{\xi_{\max }}{r} \sin \frac{\pi r}{2 \Lambda}(-1)^{[t / T]} \sin \frac{\pi t}{2 T}
\end{aligned}
$$


In this region the tension of space, as following from the solution $(21)$, is $\xi \approx 0$.

The solution (22) illustrates the availability of a deformation potential $\propto 1 / \mathrm{r}$ in the environment of the particle. In this range cells of the tessel-lattice are a little bit deformed, which induces the phenomenon of attraction, as from the distribution (22) we may obtain Newton se gravitational potential

$$
u=-G \frac{m_{0}}{r} .
$$

In the case of a missive body, the gravitation emerges owing to the bodyes entities, namely, vibrations of the entities near their equilibrium positions. The corresponding amplitudes are the entities $^{\text {ee }}$ de Broglie wavelengths. Hence a cloud of inertons covers each entity and these clouds overlap forming a unified inerton cloud of the body, which spreads far beyond the body inducing the potential (23).

Gravity must consist of two components: radial and tangential. The second is always present in a test body that is attracted by the larger central object, which was pointed out by Poincaré [11]. It was shown [12] that the correct attractive potential is slightly different from the classical Newtonian and has the form

$$
U=-G \frac{M m}{r}\left(1+\frac{r^{2} \dot{\varphi}^{2}}{c^{2}}\right)
$$

where $\mathrm{M}$ and $\mathrm{m}$ are masses of the central object and the test body, respectively, and the tangential velocity of the test body is $r \dot{\varphi}$.

Expression (24) was successfully used [12] to obtain exactly the same equations and the solutions for such problems (four classical tests), as the motion of Mercury ${ }^{\text {ee }}$ perihelion, the light deflection by the Sun, the gravitational redshift of spectral lines, and the derivation of the Shapiro time delay effect.

Expression (24) is transparent, unlike the situation with general relativity, in which gravity equations are separated from the metric that the equations contain. The submicroscopic concept does not predict the black hole solution for gravitating masses and also deny gravitons. At the same time the submicroscopic concept allows one to solve the problem of dark matter [13] and dark energy [14].

Of course inerton field effects were verified experimentally both in the microscopic and macroscopic experiments $[12,14]$. In particular, the correct determination of photons and the vibration of entities in solids made it possible to unveil the phenomenon of diffraction of light in terms of free photons (excitations of the tessel-lattice) and inertons of the vibrating entities of the interferemeter in question [15], which rejects the previous explanation based on the vague idea of wave-particle [1,2].

\section{Conclusion}

Thus the submicroscopic concept clarifies all items listed in Section 2, which so far have been neglected by all other particle theories. I has been shown that the real physical space is the tessellattice constituted of topological balls. The dynamic inerton field generated by a moving particle induces both the quantum mechanical and the gravitational interaction. Gravity is a pure dynamic phenomenon, which, however, has been perceived as a static geometry of general relativity.

The microscopic world looks complete deterministic. The de Broglie wavelength $\lambda$ is a spatial period of a moving particle. Within the section $\lambda$, due to the emission and re-absorption of the 
particle ${ }^{\text {ee }}$ inerton cloud, parameters of the particle undergo periodical changes: velocity $v_{0} \rightarrow 0 \rightarrow v_{0}$; mass $m \rightarrow 0 \rightarrow m$ and the tension $0 \rightarrow \xi \rightarrow 0$; electric charge $e \rightarrow 0 \rightarrow e$ and the magnetic charge, i.e. monopole state $0 \rightarrow g \rightarrow 0$ particle shape: beanlike $\rightarrow$ spherical $\rightarrow$ beanlike (such internal motion manifests itself in conventional quantum mechanics as a half-integral spin). In the realm of particle physics the theory allows one to operate with the kinetic of a particle inside the de Broglie wavelength $\lambda$, which is out the possibility of any other approach.

Supersymmetry predicted new particles but it failed tests. The absence of predicted sparticles in the experiment may be associated with a typical misunderstanding caused by ignorance of the submicroscopic behaviour/kinetics of particles. Indeed, supersymmetry does not consider the spin kinetics of a particle in the section of $\lambda$. But the particle is specified with three subsystems: the real particle (the particable cell), the particle sef deformation coat, and the particle se cloud of excitations. In the case of a lepton these excitations are inertons; in the case of a quark they are rather gluons. Hence a supersymmetric partner to the particle in question is rather the particle ${ }^{e e}$ components - its deformation coat and the inerton cloud (or gluon cloud for a quark).

In the future it seems it will be interesting to apply the submicroscopic concept to such challenges as: the phenomenon of confinement, the interaction between quarks through strong and weak forces, the dynamics of quarks in baryon, stability of nucleons, and the nuclear forces. The concept is able to unveil the kinetics of particles and the particle transformations and hence will open a gateway to physics beyond the Standard Model. Nevertheless, for the solution of problems associated with the spectrum of particles (i.e. their energy, or mass) quantum field theory remains preferable.

\section{References}

[1] P. S. Epstein and P. Ehrenfest, Proc. Natl. Acad. Sci. USA 10, no. 4, 133-139 (1924).

[2] P. Ehrenfest and P. S. Epstein, Proc. Natl. Acad. Sci. USA 13, no. 6, 400-408 (1927).

[3] V. S. Varadarajan, Supersymmetry for mathematicians: An introduction (Courant lecture notes; 11), American Mathematical Society, Courant Institute of Mathematical Sciences, New York; American Mathematical Society Providence, Rhode Island, 2004.

[4] J. Lykken and M. Spiropulu, Scientific American 310, no. 5, 22-27.

[5] M. Shifman, Reflections and impressionistic portrait at the conference „Frontiers Beyond the Standard Model', FTPI, Oct. 2012, arXiv:1211.0004.

[6] M. Bounias and V. Krasnoholovets, Kybernetes: The Int. J. Systems \& Cybernetics 32, no. 7/8, 945-975 (2003). Eds.: L. Feng, B. P. Gibson \& Yi Lin; arXiv:physics/0211096.

[7] M. Bounias and V. Krasnoholovets, ibid. 32, no. 7/8, 976-1004 (2003). Eds.: L. Feng, B. P. Gibson \& Yi Lin; arXiv:physics/0212004.

[8] M. Bounias and V. Krasnoholovets, ibid. 32, no. 7/8, 1005-1020 (2003). Eds.: L. Feng, B. P. Gibson \& Yi Lin; arXiv:physics/0301049.

[9] M. Bounias and V. Krasnoholovets, Int. J. Anticip. Computing Systems 16, 3-24 (2004). Ed.: D. Dubois; arXiv:physics/0309102.

[10] A. H. Compton, Phys. Rev. 21, no. 5, 483-502 (1923).

[11] H. Poincaré, Rendiconti del Circolo matematico di Palermo 21, 129-176 (1906); also: Oeuvres, t. IX, pp. 494-550.

[12] V. Krasnoholovets, in American Institute of Phys. Conference Proc. 1316, 244-268 (2010). In: Search for Fundamental Theory: The VII International Symposium Honoring French Mathematical Physicist Jean-Pierre Vigier (12-14 July 2010, Imperial College, London). Eds.: R. L. Amoroso, P. Rowlands \& S. Jeffers. 
[13] V. Krasnoholovets, Ind. J. Theor. Phys. 48, no. 2, 97-132 (2000); arXiv:quant-ph/0103110.

[14] V. Krasnoholovets, Hadronic J. Suppl. 18, no. 4, 425-456 (2003); arXiv:physics/0501132.

[15] V. Krasnoholovets, Astroph. Space Sci. 335, no. 2, 619-627 (2011).

[16] V. Krasnoholovets, in Recent developments in dark matter research, Eds.: N. Kinjo \& A. Nakajima. New York, Nova Science Publishers, 2014, pp. 1-61.

[17] V. Krasnoholovets, Nonlin. Optics, Quant. Optics 41, no. 4, 273 - 286 (2010). 